\title{
Immune defects in patients with pulmonary Mycobacterium abscessus disease without cystic fibrosis
}

\author{
Milou M.F. Schuurbiers ${ }^{1,5}$, Mariolina Bruno $\mathbb{1}^{2,5}$, Sanne M.H. Zweijpfenning ${ }^{1,5}$, \\ Cecile Magis-Escurra ${ }^{1}$, Martin Boeree ${ }^{1}$, Mihai G. Netea ${ }^{2,3}$, Jakko van Ingen $\mathbb{1}^{4}$, \\ Frank van de Veerdonk ${ }^{2}$ and Wouter Hoefsloot (1) ${ }^{1}$
}

Affiliations: ${ }^{1}$ Radboud University Medical Centre, University Centre of Chronic Diseases Dekkerswald, Dept of Pulmonary Diseases, Nijmegen, The Netherlands. ${ }^{2}$ Dept of Internal Medicine, Radboud Institute for Molecular Life Sciences, Radboud University Medical Center, Nijmegen, The Netherlands. ${ }^{3}$ Dept for Genomics and Immunoregulation, Life and Medical Sciences Institute (LIMES), University of Bonn, Bonn, Germany. ${ }^{4}$ Radboud University Medical Centre, Dept of Medical Microbiology, Nijmegen, The Netherlands. ${ }^{5}$ These authors contributed equally.

Correspondence: Sanne M.H. Zweijpfenning, Radboud University Medical Centre, University Centre of Chronic Diseases Dekkerswald, Dept of Pulmonary Diseases, P0 Box 9101, 6500HB Nijmegen, The Netherlands. E-mail: sanne.zweijpfenningQradboudumc.nl

ABSTRACT The prevalence of Mycobacterium abscessus infections in non-cystic fibrosis (CF) patients has increased in recent years. In this study, we investigate whether immune defects explain the apparent susceptibility to this opportunistic infection in non-CF patients.

We performed stimulations of peripheral blood mononuclear cells and whole blood from 13 patients with $M$. abscessus pulmonary disease and 13 healthy controls to investigate their cytokine production after $24 \mathrm{~h}$ and 7 days.

Patients were predominantly women (54\%) with a mean age of 59 years; $62 \%$ had nodular bronchiectatic disease. Many patients had predisposing pulmonary diseases, such as COPD (46\%), and asthma (23\%). Patients with COPD showed an impaired interleukin (IL)-6 response to M. abscessus and a reduced IL-17 response to Candida, together with a $M$. abscessus-specific enhanced IL-22 production. Patients without COPD showed higher levels of interleukin-1 receptor antagonist (IL-1Ra), an antiinflammatory molecule. Within the non-COPD patients, those with bronchiectasis showed defective interferon (IFN) $-\gamma$ production in response to Candida albicans.

In conclusion, susceptibility to $M$. abscessus is likely determined by a combination of immunological defects and predisposing pulmonary disease. The main defect in the innate immune response was a shift of the ratio of IL-1 $\beta$ to IL-1Ra, which decreased the bioactivity of this pathway in the adaptive immune response. In the adaptive immune response there was defective IL-17 and IFN- $\gamma$ production. Patients with COPD and bronchiectasis showed different cytokine defects. It is therefore crucial to interpret the immunological results within the clinical background of the patients tested.

@ERSpublications

Measurement of defects in both the innate and adaptive immune responses in patients with M. abscessus pulmonary disease show that susceptibility to $M$. abscessus is determined by a combination of immunological defects and predisposing pulmonary disease https://bit.ly/2DtbycY

Cite this article as: Schuurbiers MMF, Bruno M, Zweijpfenning SMH, et al. Immune defects in patients with pulmonary Mycobacterium abscessus disease without cystic fibrosis. ERJ Open Res 2020; 6: 00590-2020 [https://doi.org/10.1183/23120541.00590-2020].

This article has supplementary material available from openres.ersjournals.com.

Received: 17 Aug 2020 | Accepted: 18 Aug 2020

Copyright $\odot$ ERS 2020. This article is open access and distributed under the terms of the Creative Commons Attribution Non-Commercial Licence 4.0. 


\section{Introduction}

Mycobacterium abscessus is a nontuberculous mycobacterium notorious for causing difficult-to-treat pulmonary infections. Due to the extreme drug resistance intrinsic to these bacteria, M. abscessus has rightfully been dubbed an "antibiotic nightmare" [1]. In the last decade, an increasing prevalence of M. abscessus infections has been reported in many countries [2]. M. abscessus comprises three subspecies: M. abscessus subsp. abscessus, M. abscessus subsp. massiliense and M. abscessus subsp. bolletii. The isolation prevalence of each subspecies varies depending on geographical location $[3,4]$.

Cystic fibrosis (CF), non-CF bronchiectasis, COPD and low body mass index (BMI) are the best-known predisposing conditions for nontuberculous mycobacteria (NTM) pulmonary disease (NTM-PD) $[2,5]$. However, only a small subset of patients with these chronic structural lung diseases acquire M. abscessus infections. In these apparently immunocompetent patients, the presence of minor defects in the immune response might explain their increased susceptibility [6]. For CF patients, such subtle immune defects were found [7].

The host immune response against NTM compromises three steps. First, mononuclear phagocytes recognise the mycobacterial cell wall via Pattern Recognition Receptors, and they internalise the mycobacterium [8]. Second, mononuclear phagocytes release pro-inflammatory cytokines interleukin (IL)- $1 \beta$, tumour necrosis factor- $\alpha$ (TNF- $\alpha$ ), IL-6, IL-12 and IL-18, which activate alveolar macrophages, dendritic cells and monocyte-derived macrophages [9]. Third, the infected dendritic cells migrate to the locoregional lymph nodes to present the mycobacterial antigen to naive T-cells, which activates the polarisation of effector T-cells and the production of T-cell-derived cytokines: IFN- $\gamma$, IL-17 and IL-22 [10]. These adaptive cytokines will in turn further activate macrophages to eliminate the mycobacterium [11].

The aim of this study was to describe a cohort of non-CF patients with pulmonary M. abscessus disease and perform a prospective evaluation of their immune responses.

\section{Methods}

Recruitment and characterisation of study subjects

All patients who had at least one positive respiratory sample for M. abscessus in our Mycobacteriology Reference Laboratory database from January 1, 2010 through April 1, 2018 were reviewed. An additional search for mycobacterial disease in our local diagnosis registry was performed to complement the patient search. Patients with M. abscessus pulmonary disease according to the American Thoracic Society (ATS) diagnostic criteria and no CF were included [12]. Written informed consent was obtained from all subjects participating in this study. For the immunological study, we recruited a healthy volunteer without an immunologically relevant medical history for each patient; in total 13 patients could be tested for immunological responses. The ethical board provided a waiver for this study due to its minimally invasive character.

\section{Data collection}

Demographic and clinical data were extracted from electronic patient files. Chest computed tomography scans were reviewed by a radiologist and subsequently by a pulmonologist of our NTM reference clinic (WH, SZ).

\section{Treatment and outcome}

Treatment outcome was classified according to the NTM-NET definitions [13]. Culture conversion was defined as three consecutive negative mycobacterial cultures from respiratory samples, collected at least 4 weeks apart, during antimycobacterial treatment. The time of conversion was defined as the date of the first negative culture.

\section{Microbiology}

Isolates were identified to subspecies level by sequencing of the erm(41) and hsp65 genes. Antimicrobial susceptibility testing was performed using broth microdilution, according to the Clinical and Laboratory Standards Institute (CLSI) guidelines [14].

\section{Immunology}

Prospective patient recruitment and blood sampling

Peripheral blood was obtained from 13 patients with a (history of) M. abscessus disease and 13 healthy volunteers. EDTA plasma was obtained by venous blood sampling and used for whole blood and peripheral blood mononuclear cells (PBMCs) stimulation tests within $2 \mathrm{~h}$ after collection. Not all patients were available for immunological testing. 
Whole blood assay

Diluted Whole Blood Assays for Cytokine Responses to Innate Stimuli was performed as described previously [15]. Peripheral whole blood was diluted 1:4 in RPMI 1640 Dutch-modified culture medium (Life Technologies/Invitrogen, Breda, The Netherlands) and pipetted into a microtitre plate (48-well plate (500000), $100 \mu \mathrm{L}$ of whole blood). The following cytokine-inducing stimuli were added: heat-killed M. abscessus bacteria (means of infection (MOI) 2; 2 mycobacteria per immune cell) of the patient's own isolate $\left(1 \times 10^{6}\right.$ cells $\left.\cdot \mathrm{mL}^{-1}\right)$, M. abscessus CIP 104536 type strain $\left(1 \times 10^{6}\right.$ cells $\left.\cdot \mathrm{mL}^{-1}\right)$, Candida albicans (clinical isolate UC820) $\left(1 \times 10^{-6}\right.$ cells $\left.\cdot \mathrm{mL}^{-1}\right)$ and Aspergillus fumigatus (clinical isolate V05) $\left(1 \times 10^{-6}\right.$ cells $\left.\cdot \mathrm{mL}^{-1}\right)$. The whole blood samples were incubated at $37^{\circ} \mathrm{C}$ for $48 \mathrm{~h}$ for measurements of TNF $\alpha$, IL- $1 \beta$ and IFN- $\gamma$ production.

Isolation and stimulation of PBMCs

Human PBMCs, as described previously [16], were isolated from EDTA blood using Ficoll-Paque density gradient centrifugation (GE Healthcare, Little Chalfont, UK) and resuspended in RPMI 1640 Dutch-modified culture medium (Life Technologies/Invitrogen, Breda, The Netherlands) at a density of $5 \times 10^{6}$ cells $\cdot \mathrm{mL}^{-1}$. The isolated PBMCs $(500000 /$ well, $100 \mu \mathrm{L})$ were seeded on a round-bottom 96 -well plate (Corning Inc., Corning, NY, USA) and supplemented with a stimulus accompanied by $10 \%$ human serum in the 7 days condition and in the Aspergillus stimulation experiment. The cells were stimulated with RPMI only as a negative control, the patients' heat-killed M. abscessus $\left(1 \times 10^{6}\right.$ cells $\cdot \mathrm{mL}^{-1}$, MOI 2), M. abscessus CIP 104536 type strain $\left(1 \times 10^{6}\right.$ cells. $\mathrm{mL}^{-1}$, MOI 2), M. avium ATCC 700898 type strain $\left(1 \times 10^{6}\right.$ cells $\cdot \mathrm{mL}^{-1}$, MOI 2$)$, heat-killed A. fumigatus V05 type strain $\left(1 \times 10^{-6} \mathrm{cells} \cdot \mathrm{mL}^{-1}\right)$ and heat-killed C. albicans UC820 type strain $\left(1 \times 10^{-6}\right.$ cells $\left.\cdot \mathrm{mL}^{-1}\right)$. The cell cultures were incubated at $37^{\circ} \mathrm{C}$ for $24 \mathrm{~h}$ for subsequent measurement of the production of TNF $\alpha$, IL-1 $\beta$, interleukin-1 receptor antagonist (IL-1Ra) and IL-6, and for 7 days to detect IFN- $\gamma$, IL-17 and IL-22.

\section{Cytokine measurements}

Culture supernatants were collected at the fixed time points and frozen at $-20^{\circ} \mathrm{C}$ until cytokine assessment. Cytokine production was determined in supernatants using commercial ELISA kits for TNF $\alpha$, IL-1 $\beta$, IL-1Ra, IL-6, IL-17, IL-22 (R\&D Systems, Minneapolis, MN, USA) and IFN- $\gamma$ (Sanquin, Amsterdam, The Netherlands) following the instructions of the manufacturer. IL-1 $\beta$ to IL-1Ra ratio, as a measure of IL-1 bioactivity, was calculated by plotting the levels of IL-1 $\beta$ and IL-1Ra in response to M. abscessus stimulation.

Testing for underlying defects responsible for IFN- $\gamma$ deficiency

To find the underlying defect responsible for the IFN- $\gamma$ deficiency, we performed additional experiments by stimulating PBMCs with the two major Type 1 T-helper (Th1)-polarising cytokines, IL-12 (10 ng. $\left.\mathrm{mL}^{-1}\right)$ and IL-18 (50 ng. $\left.\mathrm{mL}^{-1}\right)$ for 7 days. In addition, using PBMCs from three patients and three controls we used either IL-12 or IL-18 separately with the addition of stimulus phytohaemagglutinin (PHA, $10 \mu \mathrm{g} \cdot \mathrm{mL}^{-1}$ ) or E. coli lipopolysaccharide (LPS, $1 \mathrm{ng} \cdot \mathrm{mL}^{-1}$ ), or a combination of both.

\section{Statistical analysis}

For the retrospective analyses, data are presented as mean (SD) for continuous variables and as number (percentage) for categorical variables. All data are analysed using SPSS version 23.0 (SPSS Inc., Chicago, IL, USA). Immunological results are presented as mean \pm SEM. Correlation analysis between clinical characteristics and cytokine secretion is performed using Spearman's rank correlation coefficient and the $95 \%$ confidence interval is calculated accordingly. A p-value of $<0.05$ is considered statistically significant. Generation of graphs and statistical analyses are performed using GraphPad Prism 5 (La Jolla, CA, USA).

\section{Results}

13 patients without CF met the diagnostic criteria for M. abscessus pulmonary disease and were included in the current study (supplementary figure S1); their baseline characteristics are shown in table 1. The predominant radiographic manifestation was nodular bronchiectatic disease $(61.5 \%)$. The baseline pulmonary function is presented in supplementary table S2. Ten patients $(76.9 \%)$ used inhalation steroids at diagnosis.

\section{Microbiology}

Five patients had M. abscessus subsp. abscessus (38.5\%); other patients had either M. abscessus subsp. boletii or M. abscessus subsp. massiliense but were not further typed. Four patients had acid-bacilli positive sputum smears (30.8\%). 


\section{TABLE 1 Baseline characteristics}

\begin{tabular}{|c|c|c|}
\hline & Patients & Healthy controls immunological study \\
\hline Subjects $n$ & 13 & 13 \\
\hline Age at time of diagnosis years & $59.2 \pm 9.4$ & $24 \pm 3.9$ \\
\hline Sex female/male & $6 / 7(46.2 / 53.8)$ & $10 / 3(77 / 23)$ \\
\hline \multicolumn{3}{|l|}{ Ethnicity } \\
\hline Caucasian & $13(100)$ & $15(94)$ \\
\hline Asian & 0 & $1(6)$ \\
\hline African & 0 & \\
\hline BMI $\mathrm{kg} \cdot \mathrm{m}^{-2}$ & $20.8 \pm 2.4$ & \\
\hline Nodular bronchiectatic & $8(61.5)$ & \\
\hline Fibrocavitary & 5 (38.5) & \\
\hline \multicolumn{3}{|l|}{ Comorbidities } \\
\hline Bronchiectasis & $5(38.5)$ & \\
\hline COPD & $6(46.2)$ & \\
\hline GOLD I & 0 & \\
\hline GOLD II & $2(30.8)$ & \\
\hline GOLD III & $4(30.8)$ & \\
\hline GOLD IV & 0 & \\
\hline Asthma & $3(23.1)$ & \\
\hline History of other NTM disease & $1(7.7)$ & \\
\hline History of tuberculosis & 0 & \\
\hline Haematological malignancy & $1(7.7)$ & \\
\hline Immunodeficiency & $2(15.4)$ & \\
\hline Diaphragmatic hernia & $2(15.4)$ & \\
\hline \multicolumn{3}{|l|}{ Other predisposing conditions } \\
\hline Smoking & $5(38.5)$ & \\
\hline History of smoking & 5 (38.5) & \\
\hline Never smoked & $3(23.1)$ & \\
\hline (History of) alcohol abuse & $3(23.1)$ & \\
\hline
\end{tabular}

\section{Antimicrobial treatment and response}

Antibiotic treatment was started in seven patients; three patients completed treatment, one patient is still on treatment, one patient discontinued treatment due to adverse effects and two patients died (supplementary figure S1). Antibiotic therapy was individualised based on drug susceptibility results and patient tolerance (supplementary figure S3). Adverse events were reported by all patients, and at least one drug was stopped because of adverse events in $86 \%$ of patients (S9).

\section{Cytokine responses}

Innate immunity: reduced bioactivity of $I L-1 \beta$

When PBMCs were stimulated with $M$. abscessus, the IL-1 $\beta$ to IL-1Ra ratio proved significantly reduced in patients (figure 1a). There was a trend in lower IL-1 $\beta$ production in patients compared to healthy controls $(\mathrm{p}=0.0721$; supplementary figure S4A). IL-1Ra production in response to $M$. abscessus was marginally higher in patients compared to controls, and it did not reach statistical significance. Interestingly, Aspergillus-induced IL-1Ra was significantly higher in patients compared to healthy controls (supplementary figure S4B). The induction in whole blood and PBMCs of TNF- $\alpha$ and IL-6 was not significantly different between patients and controls (supplementary figure S4C-D, S5C-D).

\section{Adaptive immunity: defective IL-17 and IFN- $\gamma$ production}

A significantly reduced IL-17 ( $\mathrm{p}<0.05$; figure $1 \mathrm{~b})$ and IFN- $\gamma$ production $(\mathrm{p}<0.05$; figure $1 \mathrm{c})$ in patients compared to controls was measured in PBMC in response to C. albicans stimulation. The whole blood stimulation with $C$. albicans confirmed the lower IFN- $\gamma$ production in patients $(p<0.01$; figure $1 d)$. No significant differences in IL-22 production were documented between patients and controls (supplementary figure S6A). To find the underlying defect responsible for the IFN- $\gamma$ deficiency, we performed additional experiments by stimulating PBMCs in the presence of the Th1-polarising cytokines for 7 days. A trend for reduced IFN- $\gamma$ production upon IL-12 + IL-18 stimulation ( $\mathrm{p}=0.0659)$ was 
observed in patients compared to controls (supplementary figure S7). A graphic summary of the immune defect is given in figure $2 \mathrm{a}$.

\section{COPD and bronchiectasis are correlated with different immune defects}

A high variability in cytokine production between patients was shown. Since COPD was the most frequent comorbidity in our patient cohort $(46.2 \%)$, we divided our patients into two clinical groups based on a formal diagnosis of COPD and compared the cytokine concentrations between those two groups and the healthy controls. In the innate response, COPD patients showed a lower M. abscessus-induced IL-6 production, compared to healthy controls ( $\mathrm{p}=0.0291$; figure $3 \mathrm{a}$ ), while in the non-COPD group IL-6 levels were similar to those in controls. In the non-COPD group, Candida-stimulated PBMCs produced more IL-1Ra than healthy controls after $24 \mathrm{~h}(\mathrm{p}=0.0261$; figure $3 \mathrm{~b})$. As for the adaptive cytokine response, in the non-COPD patient group a more prominent IFN- $\gamma$ deficiency in response to $C$. albicans was seen $(p<0.01$; figure 3c). On the contrary, IL-17 deficiency in response to Candida stimulation was more prominent in the COPD group (figure 3d). Also, COPD patients showed a significantly increased IL-22 production with M. abscessus (figure 3e). To characterise the patients in the non-COPD group this group is stratified based on non-COPD bronchiectasis. Non-COPD patients with bronchiectasis still showed a significant defect in IFN- $\gamma(\mathrm{p}=0.0109)$ and a trend towards higher IL-1Ra production $(\mathrm{p}=0.0552)$ compared to healthy controls
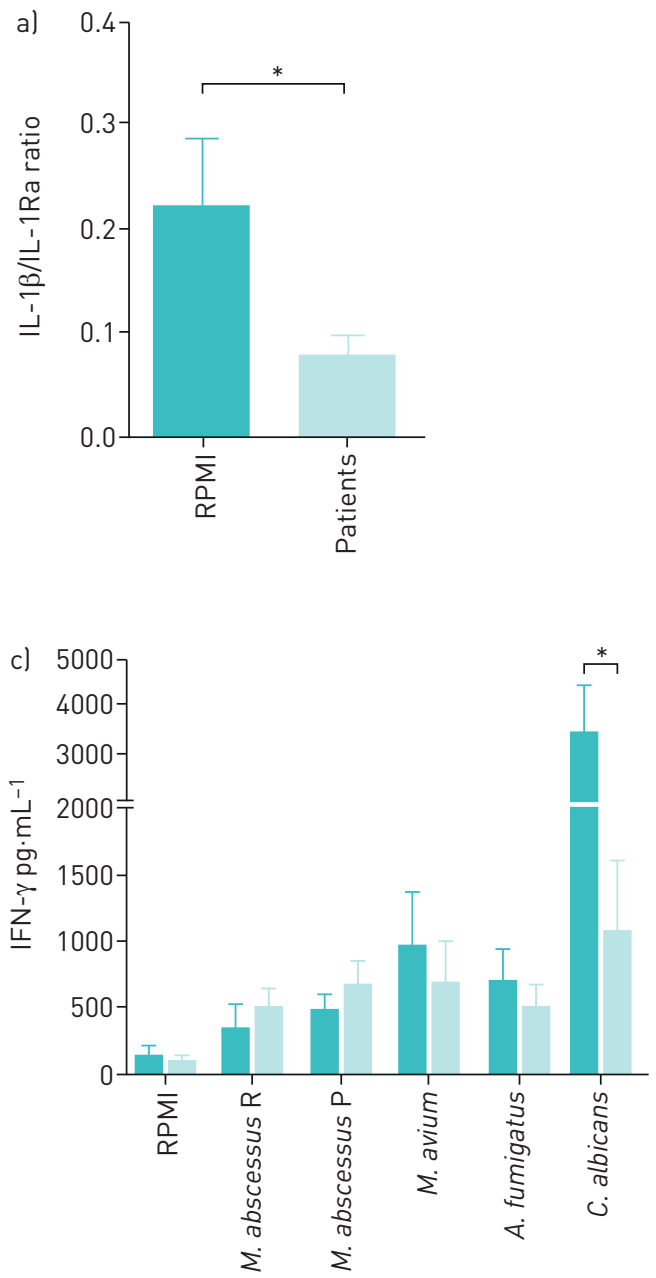
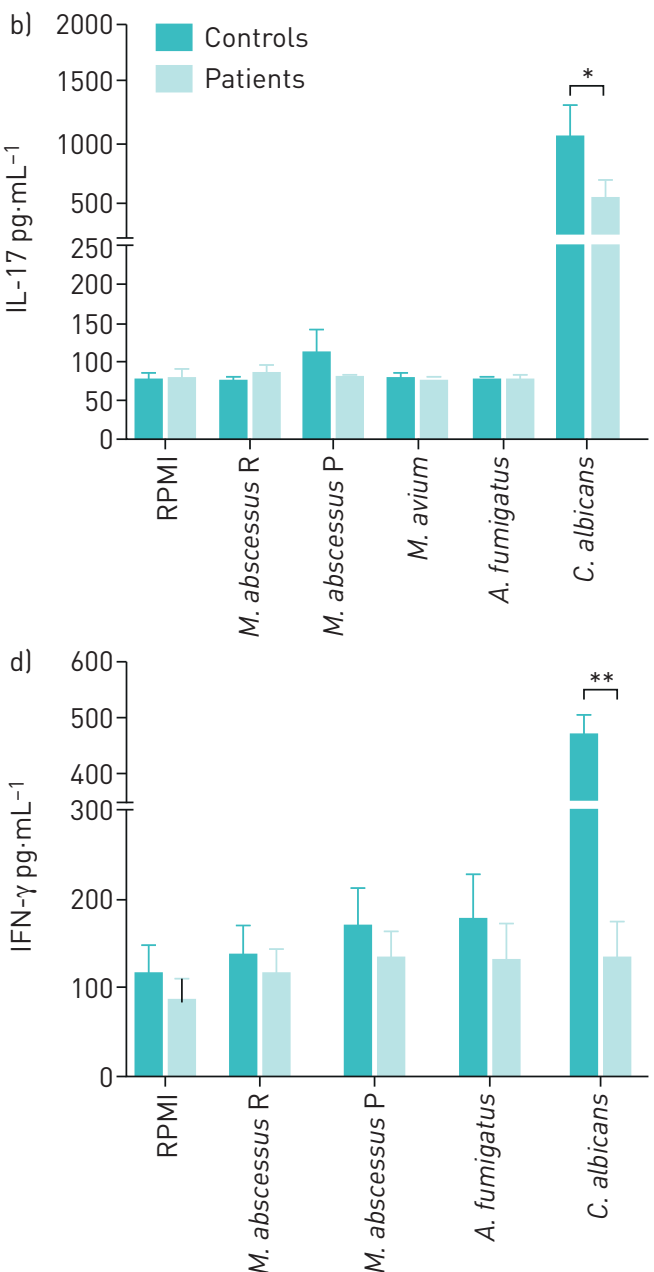

FIGURE 1 Innate and adaptive immune responses of non-cytstic fibrosis pulmonary Mycobacterium abscessus patients (n=13) and healthy controls $(n=13)$. a) Ratio of cytokine $M$. abscessus-induced production of interleukin (IL)-1 $\beta$ and interleukin-1 receptor antagonist (IL-1Ra) between patients and controls. IL-17 (b) and interferon- $\gamma($ IFN- $\gamma)(c, d)$ production upon 7 days stimulation of peripheral blood mononuclear cells $(b, c)$ or whole blood (d) with RPMI, reference Mycobacterium abscessus (clinical isolate CIP 104536) $\left(1 \times 10^{6}\right.$ cells $\left.\cdot \mathrm{mL}^{-1}\right), M$. abscessus from patient's own isolate $\left(1 \times 10^{6}\right.$ cells $\left.\cdot \mathrm{mL}^{-1}\right), M$. avium (clinical isolate ATCC 700898) $\left(1 \times 10^{6}\right.$ cells $\left.\cdot \mathrm{mL}^{-1}\right)$, Aspergillus fumigatus (clinical isolate V05) $\left(1 \times 10^{6} \mathrm{cells} \cdot \mathrm{mL}^{-1}\right)$ and Candida albicans (clinical isolate UC820) $\left(1 \times 10^{6}\right.$ cells $\left.\cdot \mathrm{mL}^{-1}\right)$. Graphs represent mean $\pm \mathrm{SEM},{ }^{*}: \mathrm{p}<0.05,{ }^{* *}$ : $\mathrm{p}<0.01$, two-tailed Mann-Whitney test. 
a)

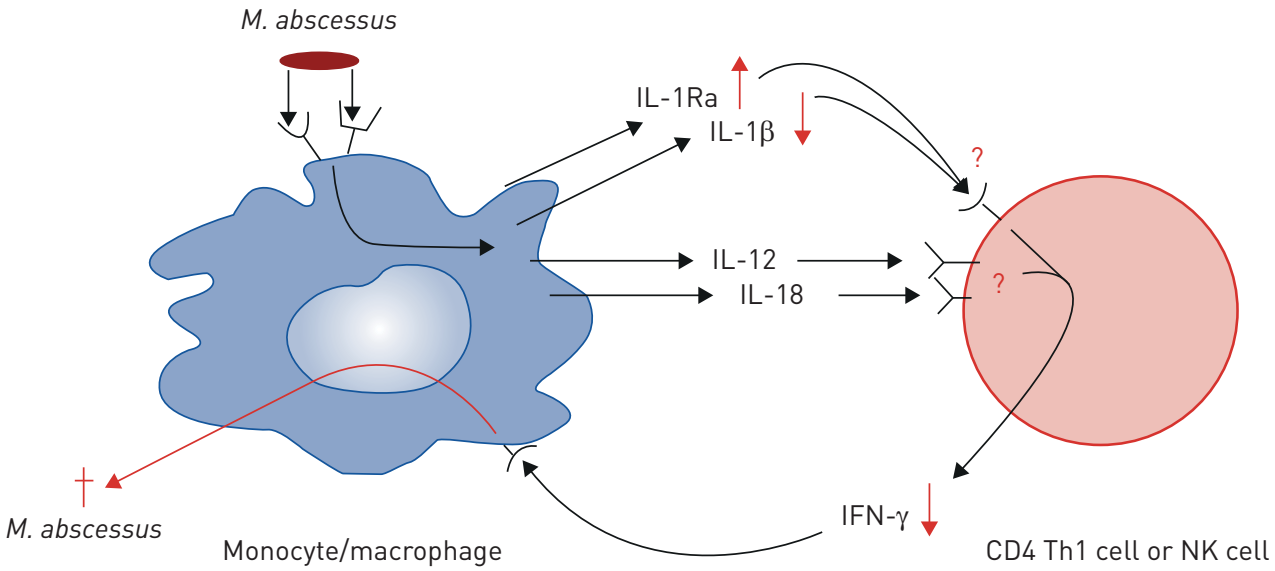

b)
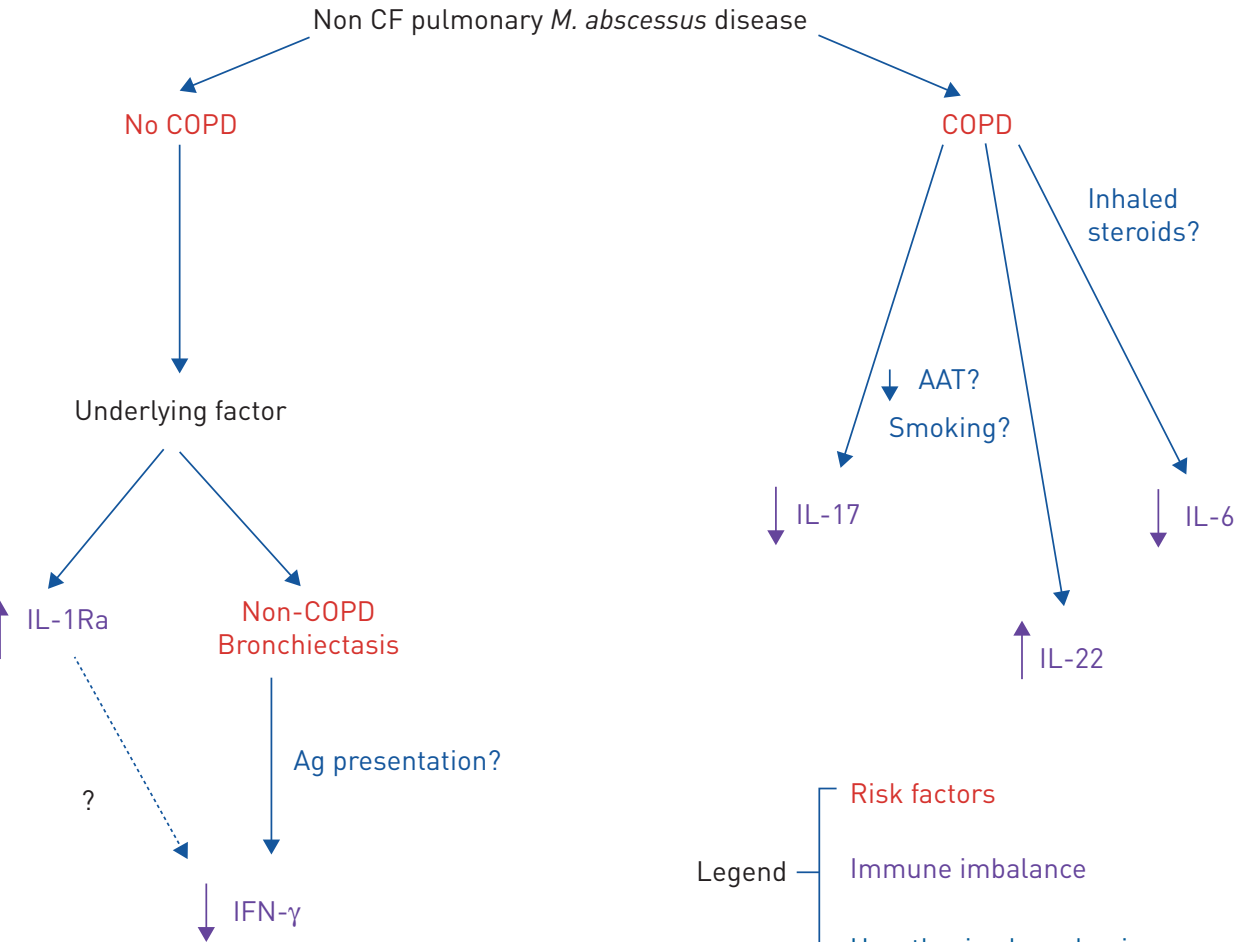

FIGURE 2 a) Overview of possible immunological defects in the immune response of non-cystic fibrosis patients with pulmonary Mycobacterium abscessus disease. b) Overview with possible underlying defects explaining the observed differences in cytokine production in chronic obstructive pulmonary disease (COPD) and non-COPD patients. IL: interleukin; Th1: Type 1 T-helper cell; NK cell: natural killer cell; AAT: $\alpha_{1}$ antitrypsin; IL-1Ra: interleukin-1 receptor antagonist; IFN- $\gamma$ : interferon- $\gamma$; Ag:antigen.

(figure $3 \mathrm{f}$ and $\mathrm{g}$ ). An overview of the above-mentioned stratification and the consequent possible immunological defect is given in figure $2 \mathrm{~b}$. To understand whether the radiological disease pattern would determine a difference in the immunological profile, we also stratified our patient cohort according to the nodular bronchiectatic disease and fibrocavitary disease type. However, except for a significantly lower M. abscessus-induced IL-6 in the nodular bronchiectasic disease as compared to healthy controls (figure $3 \mathrm{~h}$ ), no differences in immune response were seen for nodular bronchiectatic disease versus fibrocavitary disease (supplementary figure S6 B-C).

\section{Discussion}

To our knowledge, this is the first study in which assessment of cytokine responses was combined with an extensive research of clinical characteristics and treatment outcome in non-CF patients with pulmonary 

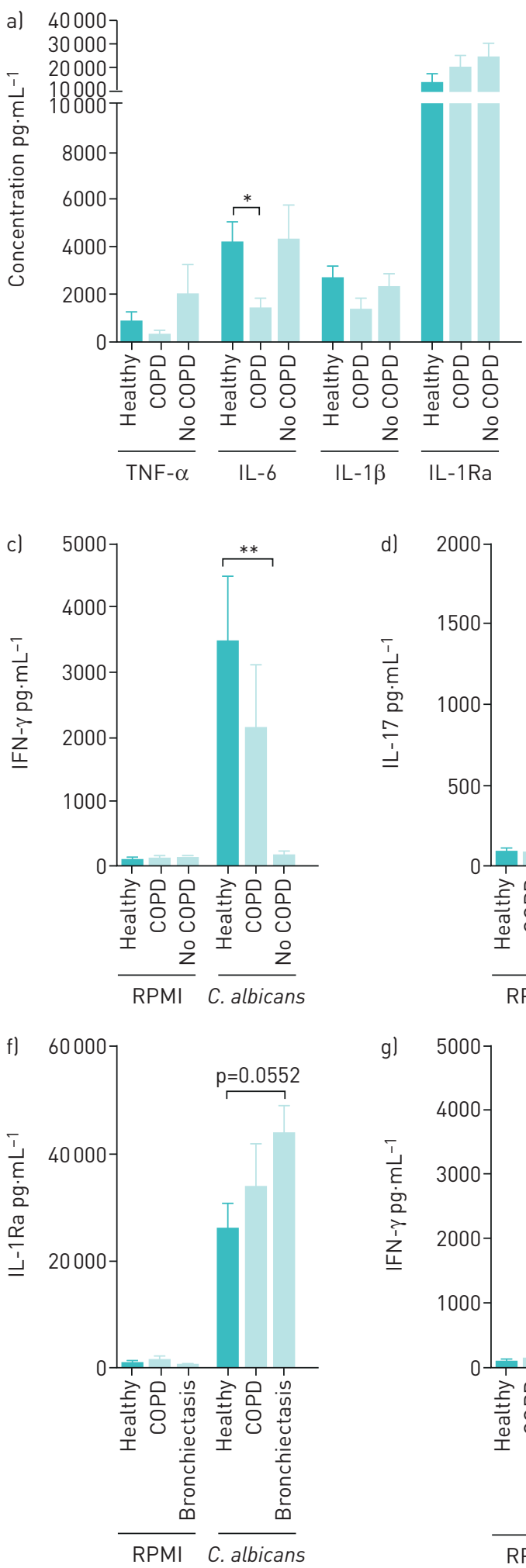
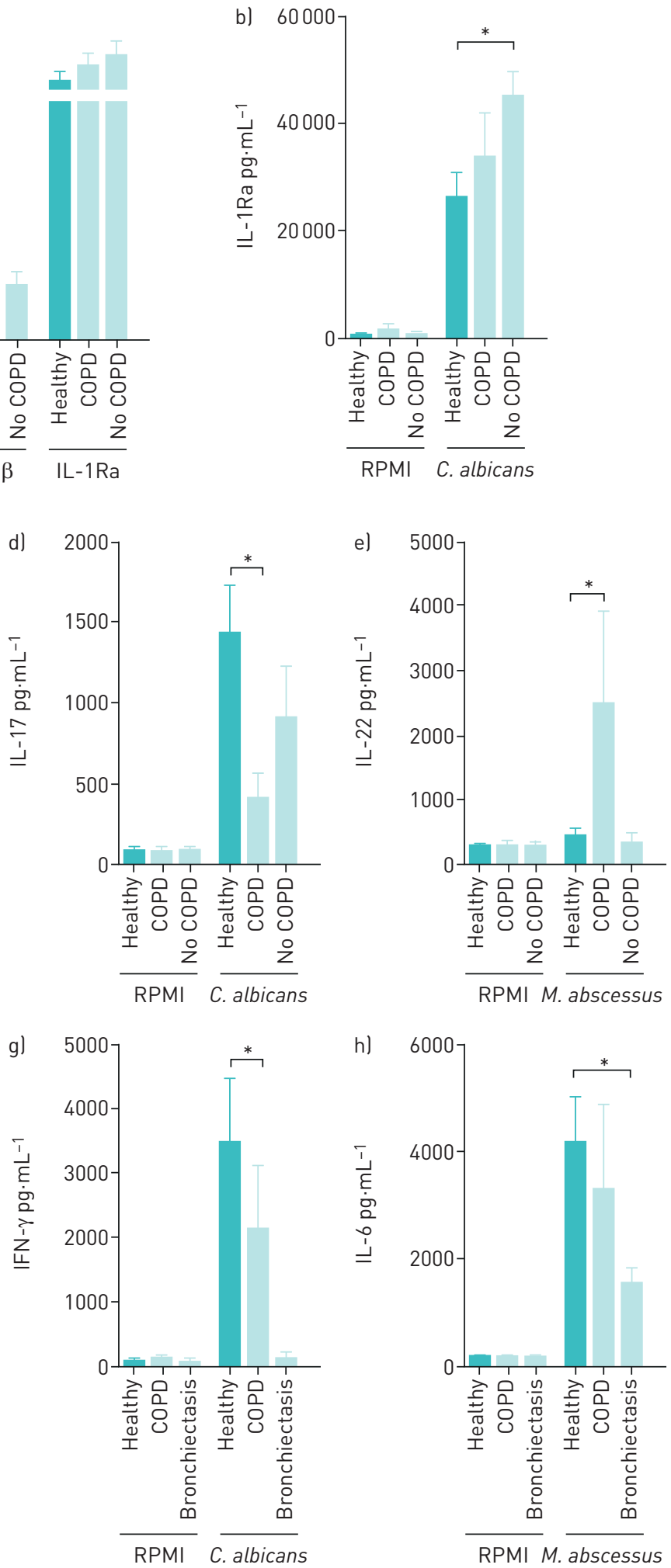

$\mathrm{R}$

FIGURE 3 Peripheral blood mononuclear cells (PBMCs) immune profile comparing different clinical subgroups. a) Innate immune cytokine production upon $24 \mathrm{~h}$ stimulation of PBMCs reference M. abscessus (clinical isolate CIP 104536) $\left(1 \times 10^{6}\right.$ cells $\cdot \mathrm{mL}^{-1}$ ); interleukin-1 receptor antagonist (IL-1Ra) (b), interferon- $\gamma$ (IFN- $\gamma$ ) (c), interleukin (IL)-17 (d) and IL-22 (e) production upon PBMC stimulation between healthy controls $(n=13)$, chronic obstructive pulmonary disease (COPD) $(n=6)$ and non-COPD ( $=7)$ patients; IL-1Ra (f) and IFN- $\gamma$ ( $g$ ) production upon PBMC stimulation between healthy controls $(n=13), \operatorname{COPD}(n=6)$ and non-COPD with bronchiectasis $(n=5)$ patients. Please note that non-COPD patients include patients with bronchiectasis; a-e $(n=5)$, since not all non-COPD patients had bronchiectasis; (h) IL-6 production upon PBMCs stimulation between healthy controls ( $n=13)$, patients with fibrocavitary disease (Cavitary, $n=4$ ) and patients with nodular bronchiectasic disease (NB, $n=8$ ). Graphs represent mean \pm SEM, *: $p<0.05$, two-tailed Mann-Whitney test. TNF- $\alpha$ : tumour necrosis factor- $\alpha$. 
M. abscessus disease. In our cohort, we found defects in both the innate immunity (a reduced bioactivity of IL-1 $\beta$ ) and in the adaptive immunity (defective IL-17 and IFN- $\gamma$ production). Clinical phenotypes such as COPD and bronchiectasis are linked with different cytokine defects.

Innate immunity: reduced bioactivity of IL-1及

In this cohort, IL-1 $1 / \mathrm{IL}-1 \mathrm{Ra}$ ratio was lower in patients than controls, which can explain a higher susceptibility to $M$. abscessus disease. The importance of IL-1 $\beta$ signalling for host response against NTM is demonstrated by the finding that activation of NLRP3 inflammasome restricts $M$. kansasii disease [17]. HAVERKAMP et al. [18] performed single nucleotide polymorphism (SNP) analysis of 81 Dutch children with NTM lymphadenitis, and they found a positive association between NTM lymphadenitis and SNPs in the IL-1 $\beta$ gene that influences IL- $1 \beta$ mRNA levels. Perhaps a lower bioactivity of $1 \mathrm{~L}-1 \beta$ could be the underlying cause of the imbalance of IL-1Ra and IL- $1 \beta$ which leads to less activation of macrophages.

\section{Adaptive immunity: defective IFN- $\gamma$ and IL-17 production}

We report a significant defect in IFN- $\gamma$ and in IL-17 production in patients compared to controls upon Candida stimulation. IFN- $\gamma$ signalling is crucial for NTM host response [19]. Mutations in receptors or transcription factors for IFN- $\gamma$ are associated with increased susceptibility to mycobacterial disease. To find the underlying defect responsible for the IFN- $\gamma$ deficiency, we performed additional experiments, but we were not able to find whether the defect was more IL-12 or IL-18 dependent (supplementary figure S7).

In our cohort deficient IL-17 production seems to be a general defect, as it is not specific to M. abscessus, but evident upon C. albicans stimulation. BECKER et al. [7] showed lower IL-22 levels while our study found higher IL-22 in COPD patients; this difference is probably due to variations in patient groups between the two studies, and the results are not significant in both studies. BECKER et al. [7] showed that IL-22 levels were lower in the non-abscessus NTM-patients, but for the M. abscessus patients with CF, levels were similar or even higher as compared to controls, similarly to our results. Since higher IL-22 levels induce lung damage and susceptibility to infection, sustained levels of IL-22 upon M. abscessus stimulation might be peculiar to patients suffering from pulmonary $M$. abscessus disease. The IL-17 defect in non-CF patients is in line with the study of BECKER et al. [7], in which an M. abscessus-specific IL-17 deficiency, rather than an IFN- $\gamma$ defect, was suggested to play a crucial role in pulmonary M. abscessus disease in patients with CF. Moreover, another study showed that the IL-23/IL-17 axis in response to $M$. avium complex (MAC) is attenuated in patients with MAC-lung disease [20]. In conclusion, NTM pulmonary disease might be associated with reduced Th17 immunity as well as with reduced Th1 immunity.

\section{Correlation with BMI}

NTM disease is associated with a certain phenotype known as Lady Windermere syndrome. Women with this syndrome typically have a low BMI, mitral valve prolapse and pectus excavatum. Also, previous studies showed that patients with pulmonary NTM disease have lower BMI and less body fat [21, 22]. When correlating IFN- $\gamma$ production in whole blood after $48 \mathrm{~h}$ with the BMI of our patient cohort, we found a significantly positive association (supplementary figure S8), meaning that patients with a lower BMI have a more pronounced IFN- $\gamma$ defect, possibly because of a defect of gamma delta-T-cells or natural killer cells [22].

\section{COPD: the great immunological divide}

We found two patterns of immune defects in two categories of $M$. abscessus patients: those with and those without COPD. Patients with COPD show an impaired IL-6 response to M. abscessus and a reduced IL-17 response to Candida, together with an M. abscessus-specific enhanced IL-22 production, which worsens lung epithelial damage. The causes of those immunological alterations might be the use of inhalation steroids, cigarette smoking or an acquired deficit of antiprotease for the excessive IL-22 response. It is known that IL-22 is significantly increased in the sputum of stable COPD [23], and the absence of IL-22 does not affect the outcome of $M$. tuberculosis infection [24]. However, a higher level of IL-22 can contribute to T-helper imbalance, and the specific IL-22 production in response to $M$. abscessus might worsen the inflammation-mediated lung damage in COPD patients. Furthermore, Jong et al. [25] also showed a defect in IL-6 production specifically for M. abscessus in COPD patients.

On the other hand, patients without COPD show higher levels of IL-1Ra, which is an anti-inflammatory molecule that blocks the bioactivity of the IL-1 pathway and might subsequently increase susceptibility to $M$. abscessus. It is suggested that reduced body fat leads to reduced leptin levels and higher levels of adiponectin, which is known to suppress the expression of TNF- $\alpha$ and induces the expression of IL-1Ra and IL-10 [5]. 
Within the non-COPD patients, those with bronchiectasis show an IFN- $\gamma$ defect in response to Candida stimulation, which might be related to impaired signalling of the polarising cytokines IL-12 and IL-18 or to the antigen presentation.

\section{Clinical characteristics}

In our cohort the high rate of COPD and the predominance of the M. abscessus subsp. massiliense stand out in comparison to cohorts from the USA, Taiwan and Japan [26-28]. The majority of patients had nodular bronchiectatic disease (61.5\%), and the number of patients with cavitary lesions in our study $(38.5 \%)$ is in line with previous studies (14-42\%) [29-31]. These aspects may partly explain the high sputum culture conversion rate in this cohort (78\%), which is similar to series from South Korea where culture conversion rates of up to $82 \%$ in $M$. abscessus subsp. massiliense pulmonary disease were observed, but only up to $26 \%$ for M. abscessus subsp. abscessus [32].

\section{Limitations}

One of the limitations of this study is the relatively small cohort: this was due to the rarity of M. abscessus pulmonary disease. A high variability between patients was observed, and larger multicentre studies will be needed to further study immunological pathways in M. abscessus patients.

In addition, it is difficult to discern whether the immune defects are causally related to M. abscessus disease or to the underlying COPD/bronchiectasis.

In conclusion, susceptibility to $M$. abscessus is probably determined by a combination of immunological defects and predisposing pulmonary disease. The main defect in the innate immune response was a shift in the ratio of IL- $1 \beta$ to IL-1Ra which decreased the bioactivity of this pathway. In the adaptive immune response we recorded a defective IL-17 and IFN- $\gamma$ production. Patients with COPD and bronchiectasis showed different cytokine defects. It is therefore crucial to interpret the immunological results within the clinical background of the patients tested. Deciphering which patients are at risk of pulmonary $M$. abscessus disease and understanding the nature of the immune response to M. abscessus in patients will hopefully enable us to develop more effective adjunctive strategies to treat this disease and potentially prevent its occurrence.

Conflict of interest: M.M.F. Schuurbiers has nothing to disclose. M. Bruno has nothing to disclose. S.M.H Zweijpfenning reports grants from Insmed and nonfinancial support from Novartis outside the submitted work. C. Magis-Escurra has nothing to disclose. M. Boeree has nothing to disclose. M.G. Netea has nothing to disclose. J. van Ingen reports advisory board membership for Insmed, Spero Therapeutics, Paratek and Janssen Pharmaceuticals outside the submitted work. F. van de Veerdonk has nothing to disclose. W. Hoefsloot has nothing to disclose.

\section{References}

1 Nessar R, Cambau E, Reyrat JM, et al. Mycobacterium abscessus: a new antibiotic nightmare. J Antimicrob Chemother 2012; 67: 810-818.

2 Cowman S, van Ingen J, Griffith DE, et al. Non-tuberculous mycobacterial pulmonary disease. Eur Respir J 2019; 11: 54.

3 Koh WJ, Jeon K, Lee NY, et al. Clinical significance of differentiation of Mycobacterium massiliense from Mycobacterium abscessus. Am J Respir Crit Care Med 2011; 183: 405-410.

4 Hoefsloot W, van Ingen J, Andrejak C, et al. The geographic diversity of nontuberculous mycobacteria isolated from pulmonary samples: an NTM-NET collaborative study. Eur Respir J 2013; 42: 1604-1613.

5 Kartalija M, Ovrutsky AR, Bryan CL, et al. Patients with nontuberculous mycobacterial lung disease exhibit unique body and immune phenotypes. Am J Respir Crit Care Med 2013; 187: 197-205.

6 Lake MA, Ambrose LR, Lipman MC, et al. 'Why me, why now?' Using clinical immunology and epidemiology to explain who gets nontuberculous mycobacterial infection. BMC Med 2016; 14: 54.

7 Becker KL, van Ingen J, Ten Oever J, et al. Deficient interleukin-17 production in response to Mycobacterium abscessus in cystic fibrosis. Eur Respir J 2016; 47: 990-993.

8 Killick KE, Cheallaigh CN, O'Farrelly C, et al. Receptor-mediated recognition of mycobacterial pathogens. Cell Microbiol 2013; 15: 1484-1495.

9 Kim TS, Kim YS, Yoo H, et al. Mycobacterium massiliense induces inflammatory responses in macrophages through Toll-like receptor 2 and c-Jun N-terminal kinase. J Clin Immunol 2014; 34: 212-223.

10 Scriba TJ, Kalsdorf B, Abrahams DA, et al. Distinct, specific IL-17- and IL-22-producing CD4+ T cell subsets contribute to the human anti-mycobacterial immune response. J Immunol 2008; 180: 1962-1970.

11 Kim SY, Koh WJ, Kim YH, et al. Importance of reciprocal balance of T cell immunity in Mycobacterium abscessus complex lung disease. PLoS One 2014; 9: e109941.

12 Griffith DE, Aksamit T, Brown-Elliott BA, et al. An official ATS/IDSA statement: diagnosis, treatment, and prevention of nontuberculous mycobacterial diseases. Am J Respir Crit Care Med 2007; 175: 367-416.

13 van Ingen J, Aksamit $\mathrm{T}$, Andrejak $\mathrm{C}$, et al. Treatment outcome definitions in nontuberculous mycobacterial pulmonary disease: an NTM-NET consensus statement. Eur Respir J 2018; 51: 1800170.

14 Clinical and Laboratory Standards Institute. Performance Standards for Antimicrobial Susceptibility Testing. Annapolis Junction, Clinical and Laboratory Standards Institute, 2018. 

innate immunity in infants identifies epidermal growth factor, IL-6, platelet-derived growth factor- $\mathrm{AB} / \mathrm{BB}$, and natural killer cell activation. Front Immunol 2017; 8: 644.

16 Bekkering S, Arts RJW, Novakovic B, et al. Metabolic induction of trained immunity through the mevalonate pathway. Cell 2018; 172: 135-146 e139.

17 Chen CC, Tsai SH, Lu CC, et al. Activation of an NLRP3 inflammasome restricts Mycobacterium kansasii infection. Plos One 2012; 7: e36292.

18 Haverkamp MH, Lindeboom JA, de Visser AW, et al. Nontuberculous mycobacterial cervicofacial lymphadenitis in children from the multicenter, randomized, controlled trial in The Netherlands: relevance of polymorphisms in candidate host immunity genes. Int J Pediatr Otorhi 2010; 74: 752-754.

19 Wu UI, Holland SM. Host susceptibility to non-tuberculous mycobacterial infections. Lancet Infect Dis 2015; 15 968-980.

20 Shu CC, Wang JY, Wu MF, et al. Interleukin 23/interleukin 17 axis activated by Mycobacterium avium complex (MAC) is attenuated in patients with MAC-lung disease. Tuberculosis (Edinb) 2018; 110: 7-14.

21 Szymanski EP, Leung JM, Fowler CJ, et al. Pulmonary nontuberculous mycobacterial infection. A multisystem, multigenic disease. Am J Respir Crit Care Med 2015; 192: 618-628.

22 Okada S, Markle JG, Deenick EK, et al. Impairment of immunity to Candida and Mycobacterium in humans with bi-allelic RORC mutations. Science 2015; 349: 606-613.

23 Zhang L, Cheng ZS, Liu WM, et al. Expression of interleukin (IL)-10, IL-17A and IL-22 in serum and sputum of stable chronic obstructive pulmonary disease patients. COPD 2013; 10: 459-465.

24 Behrends J, Renauld JC, Ehlers S, et al. IL-22 is mainly produced by IFN gamma-secreting cells but is dispensable for host protection against Mycobacterium tuberculosis infection. PLoS One 2013; 8: e57379.

25 de Jong E, Lim A, Waterer G, et al. Monocyte-derived macrophages do not explain susceptibility to pulmonary non-tuberculous mycobacterial disease. Clin Transl Immunology 2012; 1: e2.

26 Jarand J, Levin A, Zhang L, et al. Clinical and microbiologic outcomes in patients receiving treatment for Mycobacterium abscessus pulmonary disease. Clin Infect Dis 2011; 52: 565-571.

27 Tung YJ, Bittaye SO, Tsai JR, et al. Risk factors for microbiologic failure among Taiwanese adults with Mycobacterium abscessus complex pulmonary disease. J Microbiol Immunol Infect 2015; 48: 437-445.

28 van Ingen J, de Zwaan R, Dekhuijzen RPN, et al. Clinical relevance of Mycobacterium chelonae-abscessus group isolation in 95 patients. J Infection 2009; 59: 324-331.

29 Han D, Lee KS, Koh WJ, et al. Radiographic and CT findings of nontuberculous mycobacterial pulmonary infection caused by Mycobacterium abscessus. AJR Am J Roentgenol 2003; 181: 513-517.

30 Chung MJ, Lee KS, Koh WJ, et al. Thin-section CT findings of nontuberculous mycobacterial pulmonary diseases: comparison between Mycobacterium avium-intracellulare complex and Mycobacterium abscessus infection. J Korean Med Sci 2005; 20: 777-783.

31 Griffith DE, Girard WM, Wallace RJ Jr. Clinical features of pulmonary disease caused by rapidly growing mycobacteria. An analysis of 154 patients. Am Rev Respir Dis 1993; 147: 1271-1278.

32 Park J, Cho J, Lee CH, et al. Progression and treatment outcomes of lung disease caused by Mycobacterium abscessus and Mycobacterium massiliense. Clin Infect Dis 2017; 64: 301-308. 